\title{
Using Touch Interface Technology for Spatial Visualization Trainin
}

\section{Dr. Lelli Van Den Einde, University of California, San Diego}

Van Den Einde is a Lecturer with Potential Security of Employment (LPSOE) at UCSD. She teaches core undergraduate courses in Structural Engineering, is the chair of the ABET committee responsible for the continuous curricular improvement process, incorporates education innovations into courses (Peer Instruction, Project-based learning), is responsible for TA training (preparing next generation faculty), serves as faculty advisor to student organizations, hears cases of academic misconduct as a member of the Academic Integrity Review Board, and is committed to fostering a supportive environment for diverse students at UCSD by serving on the faculty advisory board for the IDEA Student Center. Her research is focused on engagement strategies for large classrooms and the development of K-16 curriculum in earthquake engineering.

Dr. Nathan Delson, University of California, San Diego 


\section{Using Touch Interface Technology for Spatial Visualization Training}

\section{$\underline{\text { Abstract }}$}

Spatial Visualization (SV) is the mental representation and manipulation of 2D and 3D shapes. Skills in SV have been correlated to high GPAs in math, engineering, computer programming, and science, yet SV is not formally part of K-12 or traditional undergraduate curriculums. A single course that teaches SV skills has been shown to increase GPA and graduation rates in Science, Technology, Engineering, and Math (STEM) fields. Studies have also shown that sketching of 3D shapes, which has traditionally been done with paper and pencil, is an important component of SV training. However, hand sketching is labor intensive to grade and students do not receive immediate feedback regarding their work. To create a more engaging and more costeffective SV training method, an interactive SV drawing application was developed at the University of California, San Diego (UCSD) using touchscreen interface technology on an iPad. The application includes an algorithm that automatically grades students' sketches and provides guidance when requested. This paper describes the development of the Spatial Visualization Trainer (SVT) and its pilot implementation at UCSD on undergraduate and high school students. Features of the SVT and areas for future work will be described.

\section{Background}

Spatial Visualization (SV) is the mental representation and manipulation of 2D and 3D shapes. Skills in SV have been demonstrated as important for success in 84 careers $^{1}$, and a 2010 report "Why So Few? Women in Science, Technology, Engineering, and Mathematics"2 identified SV as an important skill for student performance in STEM courses. The report also cited statistics that women and other underrepresented minorities (URMs) in STEM have on average lower SV skills. Moreover, the need for proficiency in SV extends beyond STEM professions. Due to the prevalence of 3D technology in data visualization and computer interactions, SV is an essential skill; yet training in this area is largely absent from most curriculums. Students with poor SV skills often find themselves at a disadvantage resulting in many of them dropping out of STEM majors. To address this disparity Sorby ${ }^{3}$ developed a short course in SV, and it has proven to effectively raise GPAs in a wide range of STEM courses among all demographics. This study at Michigan Tech showed that only $42 \%$ of students majoring in engineering with low SV skills graduated in their major. However, through the implementation of a single class in SV training, the retention rate of students with low SV skills increased to $64 \%$ (an increase of $52 \%$ ). The overall GPA for students at risk from dropping their engineering major increased from 2.64 to 3.00 .

The strength of this data has led the National Science Foundation (NSF) to create the ENGAGE program that has supported SV courses at 30 universities starting in 2010 . However, this is just a small fraction of the universities in the United States. Accordingly, there is a need to make SV education available to a wide audience (beginning in K-12 education) at low cost. 
Interest in SV skill development stems from the strength of the evidence of its effectiveness. Sorby ${ }^{5}$ performed studies where incoming engineering students took an SV assessment exam, and those that failed were required to take a one unit course on SV skill development. An example SV question from a standardized Purdue SV Assessment Exam typically given to students as a pre-test is shown in Figure 1. A comparison was made between the group that was required to take the SV course and those that barely passed the exam. The results showed that the SV course increased retention rates and student GPA in math, engineering, and even computer science above the performance of the students who initially had slightly higher SV scores but did not take the course. This study had no selection bias since the criteria for taking the course was objective, and thus conclusively shows that a single one-unit course in SV is effective at improving student performance.
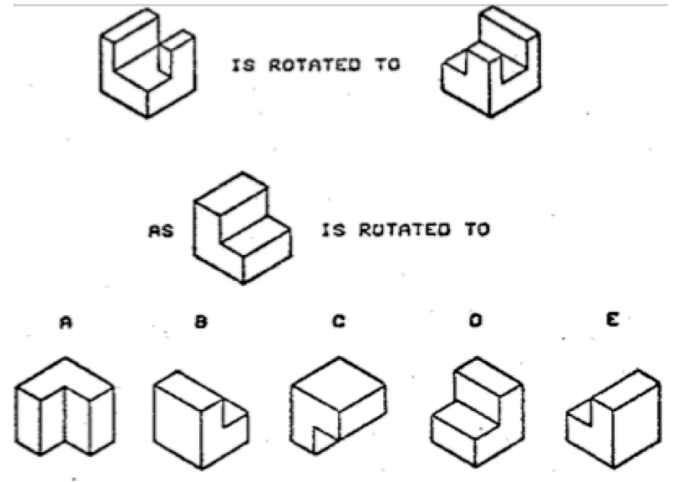

Figure 1: Example from Standardized SV Test

The ENGAGE ${ }^{4}$ program has already compiled a significant amount of curriculum for SV skill development, and provides a recommended method for teaching SV skills that is led by a teacher in the classroom and includes components from a workbook and online multimedia program 6:

- The use of multiple-choice questions and paper and pencil sketching exercises of isometric (3D) shapes and 2D orthographic projections using the Developing Spatial Thinking (DST) workbook (by Sorby)

- Explanatory material and accompanying animations provided by the DST software (Wysocki) ${ }^{6}$

However, a critical component missing from the multimedia program is the capability for hand sketching, which was revealed by Sorby ${ }^{3}$ to be a significant factor in spatial skills development. Despite the low effectiveness of using only the multimedia software for SV training, Sorby's study showed that students prefer this medium ${ }^{3}$. Sorby's multimedia program was developed when mouse and keyboard input were the only widely available computer interfaces. However, with the popularity of tablet computers, stylus and fingertip input allow for the addition of hand sketching to SV training, ultimately providing an engaging environment for the students. This new opportunity with tablet computers is the foundation of the work described in this paper.

Touch screen interfaces have only recently become widely used, and their full potential has not yet been realized. The revolutionary iPhone, which debuted in 2007, introduced the touch interface primarily for navigation. The iPad that followed in 2010 transformed the computer industry, empowering new applications where users can create drawings and other content by interacting with the large screen. Tactile interaction with a touch screen can provide high fidelity input not possible with a computer mouse making SV skill development a natural fit with this technology. Therefore, to create a more engaging and more cost-effective SV training method, an interactive SV drawing application was developed at UCSD using touchscreen interface technology on an iPad. The application includes an algorithm that automatically grades students' 
sketches and provides guidance when requested. The Spatial Visualization Trainer (SVT) was piloted on undergraduate and high school students to investigate if the immersive and tactile format is effective in increasing SV skills, which will ultimately enable increased recruitment and retention of engineering students from all demographics.

\section{SVT Sketching App}

Initially, the touch interface of the iPad that was introduced in 2010 was focused on menu navigation similar to the iPhone. However, the larger screen of the iPad allowed for more content creation, and users began taking notes and drawing on tablet computers. It turns out that the touch screen interface is ideal for SV training. Students can interact with virtual 3D shapes using their finger to rotate, zoom-in, or drag objects, and instructional animations can be integrated into various applications. The SVT application that was developed at UCSD incorporates all of these features and allows for a more automated sketching capability to enhance SV training as shown in Figure 2 (a video demonstrating these features is available ${ }^{7}$ ).

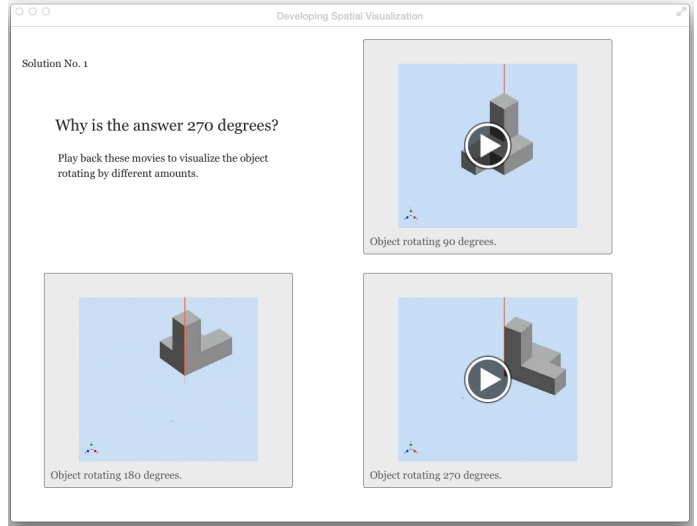

a) Animation illustrations

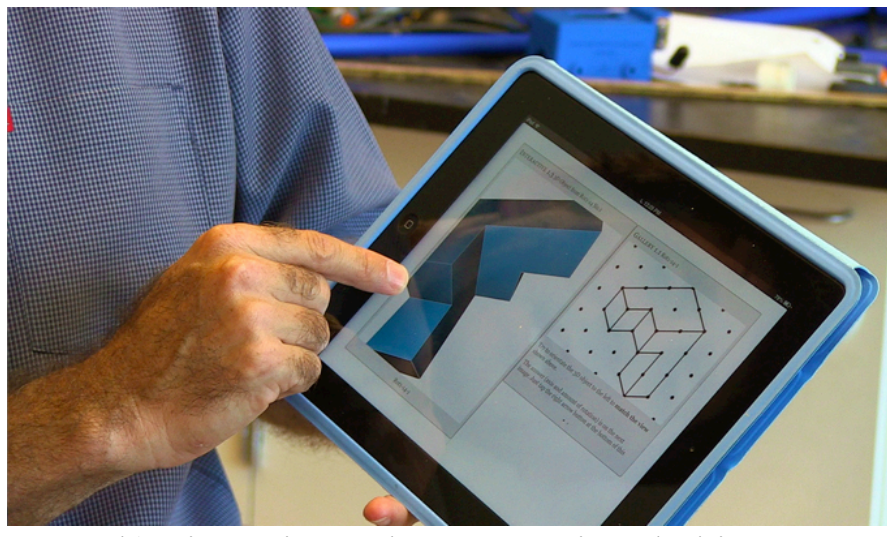

b) Finger is used to rotate virtual object

Figure 2: Touch Screen Used for Interactive SV Training

The SVT app creates a grid in a small assignment window and a corresponding grid on a larger sketching window. Both the assignment and sketching windows have a designated starting dot and the user must begin his sketches such that the starting dots and the grid match between the assignment and sketching windows. The SVT app interface is very simple with a single color pen tool, an eraser, and the ability to clear all sketch lines by shaking the iPad. Figure 3 shows both the assignment and sketching windows to demonstrate the simple exercise of drawing a cube.

One of the key features of the SVT app is the automated grading algorithm, formulated at UCSD, that provides immediate feedback to the user ultimately guiding them through the solutions, and relieves the teacher from monotonous grading. Therefore, the SV training using the SVT iPad App can be completed self-paced with minimal instructor supervision. Since the sketching assignments have a designated starting dot, there is a unique solution relative to the grid for each assignment. The grading algorithm determines if a user's sketch submission is the proper solution for the assignment within a designated tolerance. Details of the algorithm will be published in a future publication. 


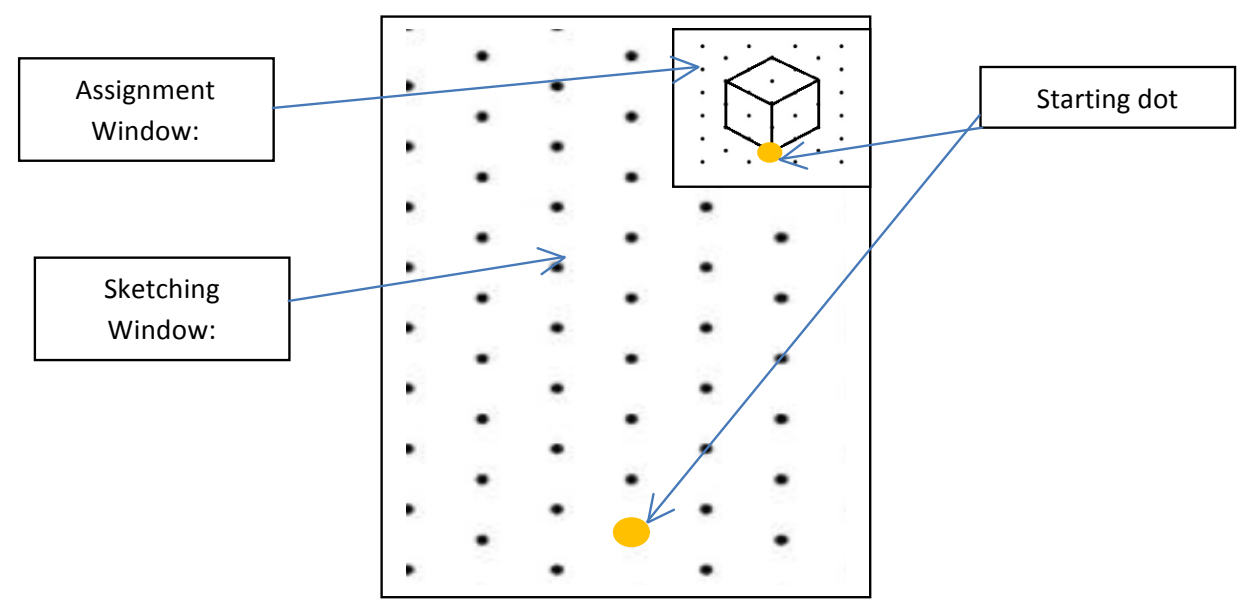

Figure 3: Assignment and Sketching Windows

Once the user completes their sketch they press the "submit" button to have their work graded. If the sketch is correct, a "good job" message is displayed to the user who moves on to the next assignment. However, if an error is detected, the user has the option to try again or peek at the solution. With a peek, the user sees the solution temporarily, but the solution disappears when the user completes their sketch. Each time a user submits a sketch, a file is sent to a server that includes a copy of the sketch, how many attempts were made, and if peeks occurred. The server that contains the student work and scores is password protected and is accessible to only the teacher and researchers. Figure 4 shows an example of a correct and incorrect sketch.

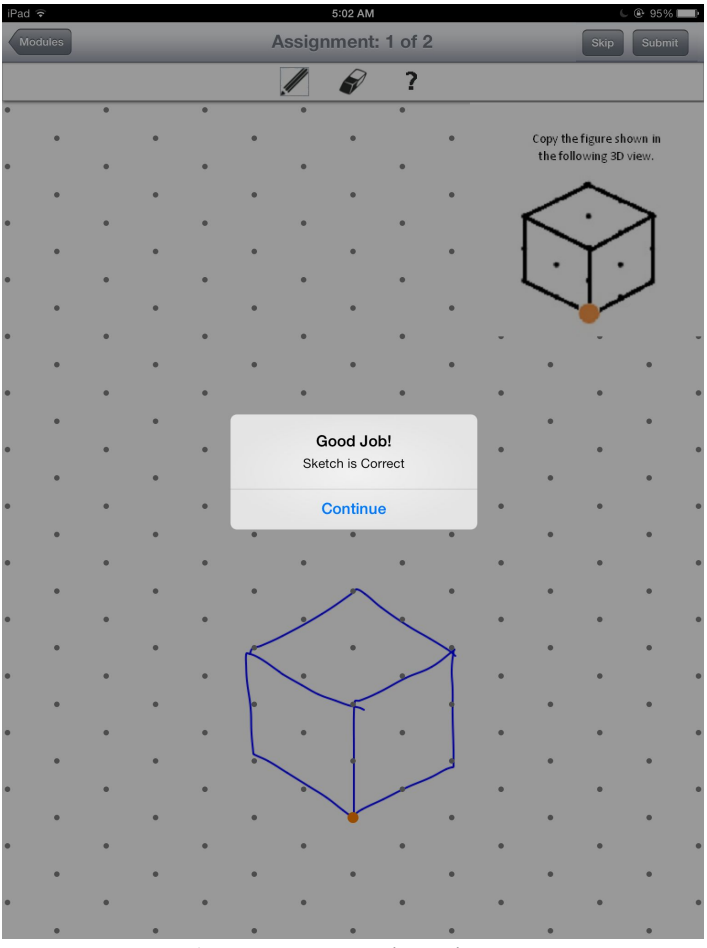

a) Correct Sketch

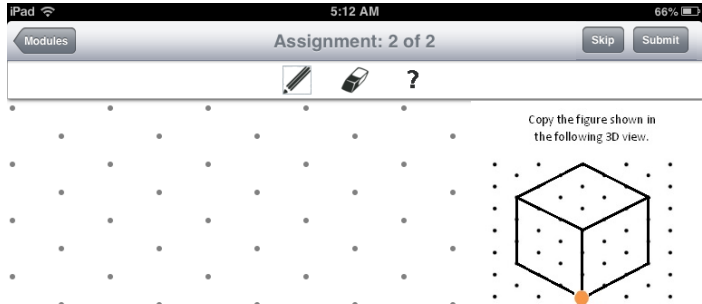

b) Incorrect Sketch with Peek at Solution (green line)

Figure 4: Automated Grading 
The sketching assignments used in the SVT app come directly from the Sorby workbook ${ }^{3}$ as per a limited 2 year trial agreement with the publisher, Cengage Learning. Of the ten modules in the workbook, listed below, only the six with asterisks (designating the modules that included sketching assignments) were incorporated directly into the SVT app.

1. Surfaces and Solids of Revolution

2. Combining Solid Objects

3. *Isometric Drawing \& Coded Plans

4. *Orthographic Drawings

5. *Inclined and Curved Surfaces

6. Flat Patterns

7. *Rotation of Objects About a Single Axis

8. *Rotation of Objects About Two or More Axes

9. *Object Reflections and Symmetry

10. Cutting Planes and Cross Sections

Since a significant number of assignments are published in the workbook, to reduce the requirement of digitizing all of the assignments and solutions in order to integrate them into the SVT app, only the sketching assignments that were assigned in the sample course on the Engage Engineering site were used.

Modules 4 and 5 require the users to learn how to draw orthographic projections, which are 2D views of an object. Since each assignment had three views to draw (top, front, and side), the drawing window for orthographic assignments provides three starting dots as shown in Figure 5. Engineering standards specify that dashed lines be drawn in orthographic projections where there is a hidden line. At this time, the grading algorithm does not grade hidden lines, and thus only orthographic assignments with solutions that did not have hidden lines were included. Grading of dashed lines will be implemented in a future version of the software.

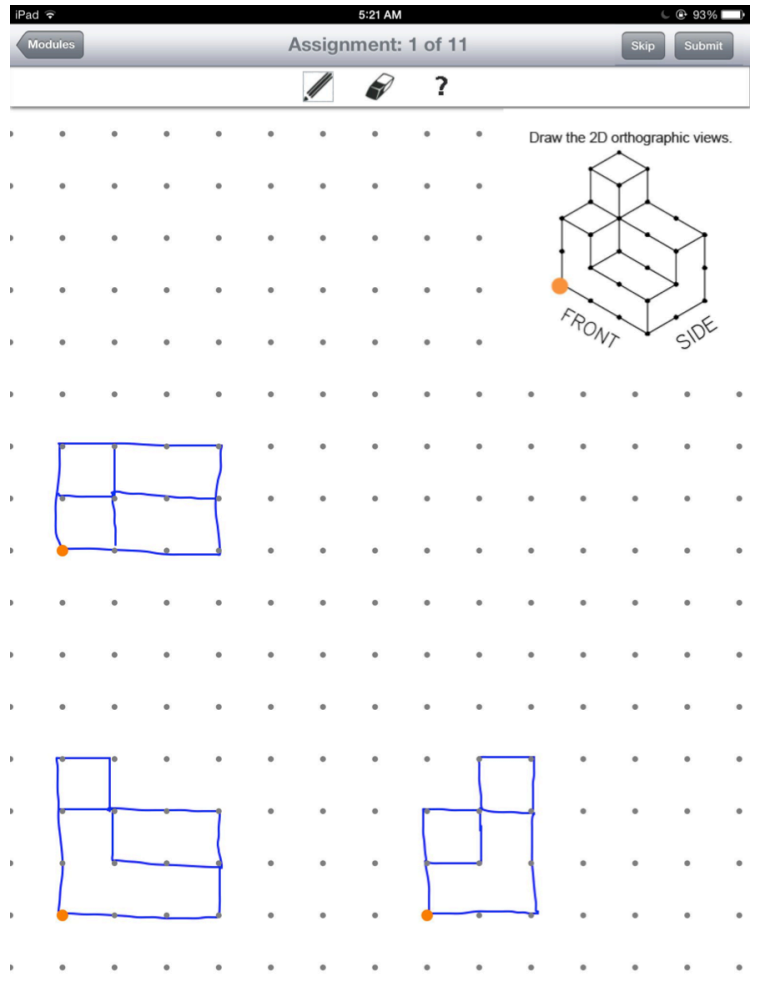

Figure 5: Orthographic Assignment with a Starting Dot in Each View

\section{$\underline{\text { Pilot Studies }}$}

In 2013, two pilot studies were implemented. Both were preliminary assessments since the SVT software was still under development. These studies were reviewed and approved to be in compliance with federal regulations regarding the protection of human subjects (UCSD IRB Project \#130252SX). 
The first pilot study was administered during spring 2013 to a small group of UCSD undergraduate students to see if hand drawing on an interactive electronic interface (iPads) would be as effective as drawing with pencil and paper. To identify potential student participants, the Purdue Spatial Visualization Test: Rotations (PSVT:R) was administered to over 1000 students in beginning mechanical and structural engineering courses. Initially, students receiving a non-passing score of $60 \%$ or less were encouraged to attend the seminar. Ultimately the seminar was opened up to any student from these courses wanting more SV training in order to increase participation. In the end, 17 students participated. Students were separated in multiple sections and attended a 10-week seminar. Approximately half the students received standard Engage Engineering instruction consisting of short lectures by a professor, the use of the DST software, and multiple choice and hand sketching exercises from the DST workbook using paper and pencil. The other half replaced the hand sketching activities with the use of the SVT application on the iPad. All instruction in both groups occurred in the classroom with no homework assigned. Student scores where recorded for all assignments and an SV test (PSVT:R) was administered on the first and last day of classes. A survey of the student experience was also conducted.

While the sample size of students participating did not result in conclusive data, students were able to use of the iPad technology without a problem and felt that the additional SV training was beneficial. At the time of this study the automated sketching algorithm was not yet completed, so the benefit of using tablets was not available to the students. However, this trial did serve to identify areas for software improvement such as navigation and implementation of the eraser tool. Some challenges that the pilot study identified were related to recruiting students and scheduling training sections. More than 50 students were originally interested in participating, but due to varied student class schedules only approximately $40 \%$ of the students could actually take the seminar. This indicated that more students would participate if they could work independently and not have the schedule constraints of a class meeting time. Another lesson learned from the pilot study was that students who needed additional SV training were less likely to volunteer to take a no-credit seminar. Most of the students who volunteered their time were strong students who were trying to get an edge compared to their fellow engineers. By in large, the students who completed the first week of the seminar ultimately completed the entire course and received a certificate of completion.

During the summer of 2013, the automated grading algorithm was implemented, and a second trial was done with a group of 23 high school students as part of the COSMOS (California State Summer School for Mathematics and Science) program, which is a 4-week residential summer program for science, technology, engineering and mathematics (STEM). The SV training was implemented in addition to an already full engineering curriculum. Students were loaned iPads to take back to the dorms with them and were given remote access to the DST software in order to interact with the tutorials at their convenience. They were provided with a short 30-minute introduction to the SVT app and the importance of SV skill development in the classroom. Otherwise, the students performed all other training independently and no grade incentive was provided to them. During this trial, only 3 out of the 10 modules in the SVT app incorporated the automated grading feature so students were asked to complete these three at a minimum. None of the multiple-choice questions from the DST workbook were used due to time constraints. The 
standardized SV test (PSVT:R) was administered at both the beginning and end of the three week period. Figure 6 shows some of the students from this high school pilot study using the SVT application.

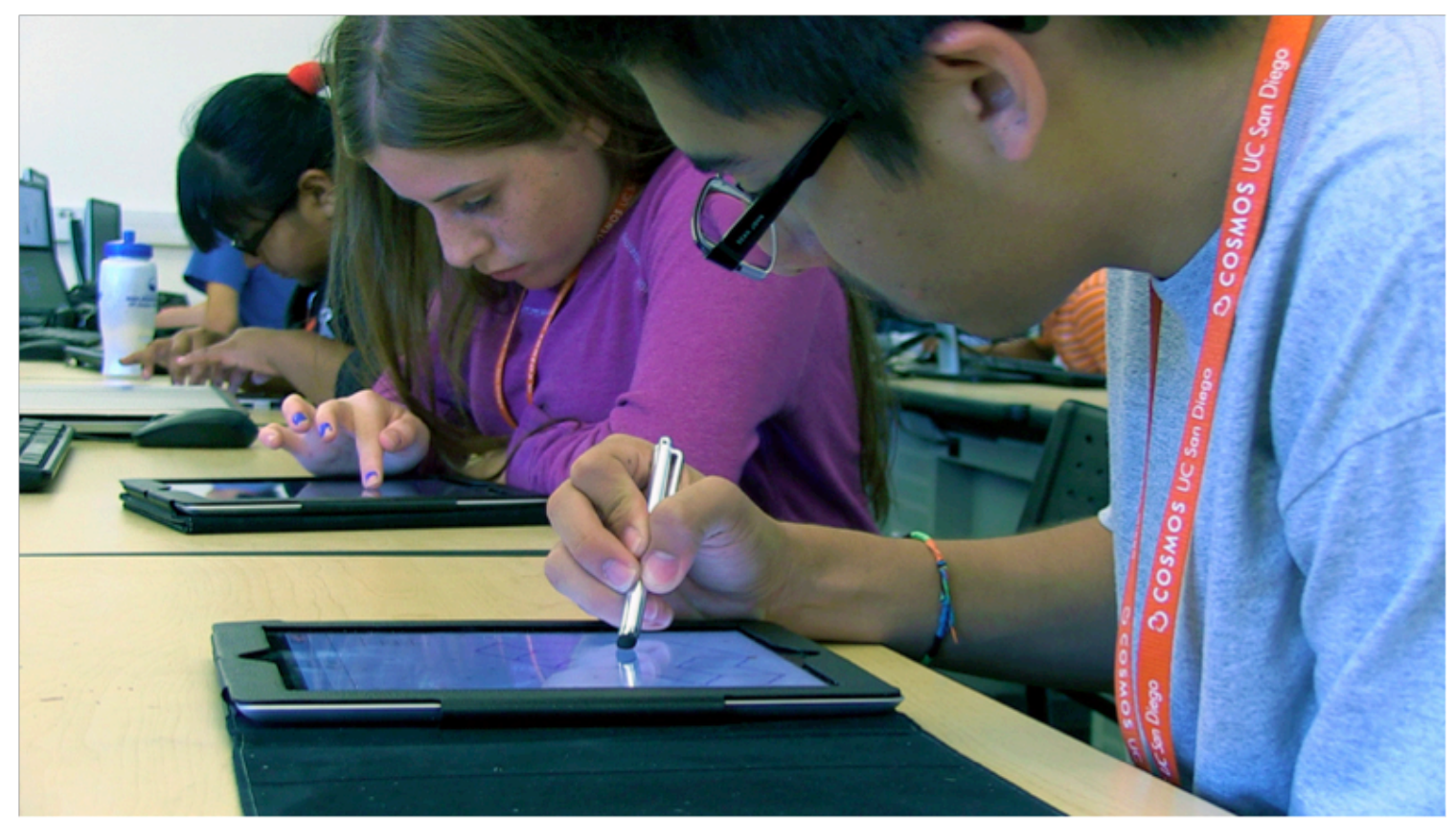

Figure 6: High School Students Sketching with the SVT App (Using Stylus or Finger)

Based on the SV Pre-Test the students were separated into three groups:

1. High Level: Students scoring $90 \%$ or higher

2. Mid Level: Students scoring between $70 \%$ and $90 \%$

3. Low Level: Students who scored at $70 \%$ or lower

The Low Level would be the typical target audience who could benefit most from SV training. Engage Engineering recommends that students who score below $60 \%$ on the PSVT:R take the class and evidence from recent research indicates that students who score between $60 \%-70 \%$ on the PSVT:R benefit from the training as well ${ }^{3}$ ). All three groups saw an increase in their SV scores between the pre-test and the post-test (refer to Table 1).

Table 1: Increase in SV Scores

\begin{tabular}{||l|c||}
\hline \multicolumn{1}{|c|}{ Group } & Increase in SV Score \\
\hline \hline High Initial Pre-Test $(n=7)$ & $1 \%$ \\
\hline Mid. Initial Pre-Test $(n=5)$ & $7 \%$ \\
\hline Low Initial Pre-Test $(n=11)$ & $16 \%$ \\
\hline All groups combined $(n=23)$ & $9 \%$ \\
\hline
\end{tabular}

The Low Level had the highest increase of $16 \%$, and 6 out of the 11 students increased to the point where they were no longer in the Low Level. The average increase for all students was 9\%. The small amount of increase in the High Level is expected since some students where so 
close to scoring $100 \%$ on the pre-test it was impossible for their score to increase even if their SV skills increased. The Low Level group representing the target audience for the SVT app was studied in more depth. In general, time spent on working with the SVT app correlated to higher SV scores as shown in Figure 7. There was one student who had a decrease in SV score even though they attempted a large number of sketching assignments, which is circled in red on the plot.

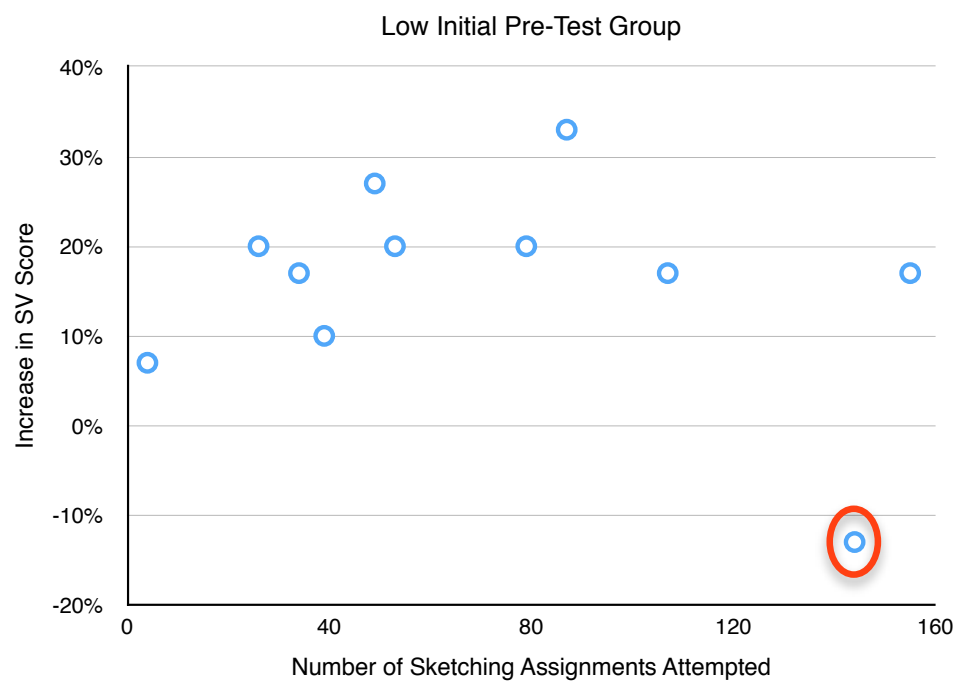

Figure 7: Increase in SV score for Low Level Group Relative to Assignments Attempted

The trend for increase in SV score is even stronger when plotted relative to the number of sketching assignments passed, as shown in Figure 8. The outlier here is even more striking since that student passed more assignments than any other.

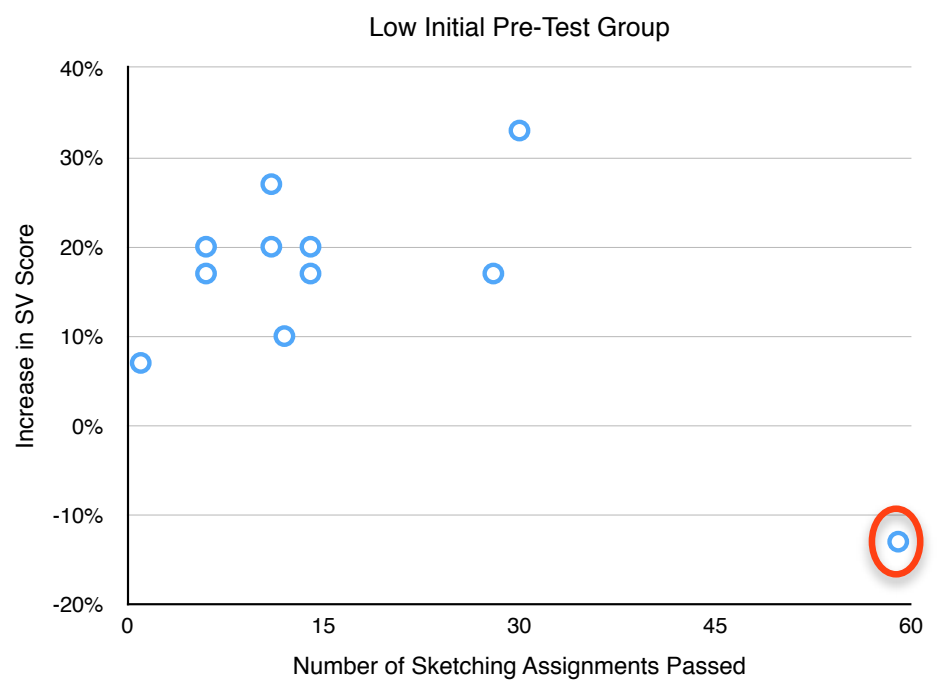

Figure 8: Increase in SV score for Low Level Group Relative to Assignments Passed 
The one outlier to the positive trend was one student who scored $60 \%$ on the pre-test. The student attempted 144 assignments and succeeded in 59 assignments, which was among the highest in the group. However the student's post-test was $13 \%$ lower than the pre-test. Interestingly, there was one student in the first pilot study with undergraduates who also saw a reduction in their test scores. The cause of these outliers is still unknown. However, in future trials the SVT app will incorporate improved tracking capabilities in order to better identify areas where students are having problems and intervene with instructor assistance for such cases.

All results at this point are preliminary due to the low number of students participating in the trials. However, one key observation is that in both pilot studies, the students continued to use the SVT app to improve their SV skills despite the fact that the SV training was not required and no grade incentive was offered. The high school students, who used the program with the automated grading algorithm, demonstrated a high level of engagement. One student noted that the app was "like playing a game."

The preliminary results are promising. In the high school group, the students were required to do only a subset of the app exercises and they were not required to do any of the multiple choice workbook questions. $30 \%$ of the high school students did not originally pass the standardized SV test (PSVT:R) pre-test (scored less than $60 \%$ ) and $17 \%$ of them were in the $60-70 \%$ range. Nevertheless, the results showed a statistically significant increase in their SV skills after using the SVT app with only $13 \%$ not passing the post-test and $9 \%$ in the $60-70 \%$ range, a $30 \%$ increase in passing rate.

The undergraduate student group also increased their average SV test score. However, most of the students who signed up for the workshop did not really need the course. Only three low scoring students participated in the workshop. Accordingly, it is recommended that incoming students be tested with the SV pre-test, and low scoring students be required to take a 1-unit SV training course.

Future Plans

Future plans include numerous improvements to the SVT app and the educational content such as:

- Improved SVT user interface and feature enhancements.

- Integrating all 10 modules into the SVT app with automated grading capabilities.

- Adding hidden line grading for orthographic projections.

- Adding a Free Sketching option where students can sketch any shape they desire and export the sketches for future use. With this tool students will be able to use a background grid to improve their sketches and remove the grid for a nicer output. Measuring how much the Free Sketching option is used may help evaluate how engaged students are in sketching.

- Developing an integrated package that includes the instructional material, multiple-choice questions, and sketching.

- Porting the software to a broader array of touchscreen devices such as Android, Windows 8 , and Chrome OS. 
- Developing a teacher interface easily enabling them to track the progress of each student, and identify where additional instruction is needed on a case-by-case basis.

- Developing a database that highlights specific assignments where students have difficulty and identifies mistakes that are repeated often to guide instructional material development.

- Developing SV content that is relevant to specific subject matter such as K-12, vocational training, molecular shapes for chemists, or human organs for medical students.

The ultimate goal of the effort described herein is to make the SVT app widely available. The software could be used in a stand-alone mode where students work independently, in a hybrid mode where teachers guide the students in a classroom, or in a mode where teaching assistants hold individual tutoring sessions for students requiring specific assistance.

The advantages of learning to sketch extend beyond improving SV skills as demonstrated on standardized tests. Sketching can improve creativity and is critical in team collaborations. In addition to the multiple choice SV tests, to document improvement in sketching ability future trials will incorporate sketching pre- and post-tests to assess how well students using the SVT app improve their sketching capabilities.

\section{Summary}

A software application to improve spatial visualization training and the skills necessary to sketch 2D and 3D shapes was successfully developed and implemented in two pilot studies. The SVT app incorporated an automated grading algorithm that provided immediate feedback to the user and guidance when needed. Feedback from users in the pilot studies indicated that they enjoyed working with the app, that the automated grading was very helpful, and that the exercises where "like a game." Even though the software used in the pilot studies did not have all modules implemented, student performance on a standardized SV test improved. These preliminary results provide a strong incentive to further develop the tool such that all components of the SV training program (instruction, videos and animations, multiple choice assignments, and sketching) are fully integrated in a package that is widely deployable.

Recent efforts in on-line education ${ }^{8}$ have not produced consistently positive results for a wide range of reasons. However, SV training is especially well suited for computer assisted learning. SV training is highly task based, and the computer makes SV training more interactive. Thanks to computer gaming, computer technology is especially strong in representing and manipulating 3D images. Based upon the preliminary results of this study that show endorsement and achievement by the participating students, the authors believe that spatial visualization training using the SVT app may become a shining example for effective use of computers in education.

\section{Acknowledgment}

The authors would like to acknowledge the Qualcomm Institute and Engaging Students in Engineering (ENGAGE) for their financial support for the development of the Spatial Visualization Trainer (SVT) and corresponding studies. Specifically, Deborah Forster, Jessica Block, Jurgen Schultz and Philip Weber (Qualcomm Institute) for program oversight, study guidance and software development; Susan Metz and Sheryl Sorby (ENGAGE) for overall 
guidance in the study; and Christina Nilles, Daneesha Kenyon, Alvin Chak, and Kane McKinnis (UCSD Students) for providing assistance in the development of the SVT digital curriculum and for serving as teaching assistants during the pilot studies

\section{References:}

1. Smith, I.M. (1964). Spatial Ability: Its Educational and Social Significance, University of London Press.

2. "Why so Few? Women in Science, Technology, Engineering and Mathematics". Published by AAUW, ISBN: 978-1-879922-40-2, 2010.

3. Sorby, S. A., \& Veurink, N. (2010). Long-term Results from Spatial Skills Intervention among First-Year Engineering Students. In Proceedings of the 65th Midyear Meeting of the Engineering Design Graphics Division of ASEE.

4. ENGAGE: an NSF funded program for engaging students in engineering; http://www.engageengineering.org/

5. Sorby, S. A. (2009). "Educational Research in Developing 3-D Spatial Skills for Engineering Students". International Journal of Science Education. (31)3.

6. Sorby, S.A. (2011). Developing Spatial Thinking Workbook and accompanying Developing Spatial Thinking Software by Anne Frances Wysocki, Cengage Learning, ISBN-13:9781111139063.

7. Overview and video of the SVT Sketching App (https://sites.google.com/a/eng.ucsd.edu/spatialvis/)

8. Kolowich, S. (2013). The MOOC 'Revolution' May Not Be as Disruptive as Some Had Imagined. Chronicle of Higher Education. 